\title{
Analysis of Glass Vial Interior Surfaces in Parenteral Drug Stability Studies
}

\author{
R. A. Carlton
}

GlaxoSmithKline, Collegeville, Pa, 19426

Most injectable pharmaceutical products (parenterals) are packaged in glass vials or syringes. Glass is an ideal packaging material due to its chemical compatibility with most parenteral products. There are, however, some parenteral products that can induce or accelerate glass corrosion processes [1]. Glass corrosion can result in the generation of glass related particulates in the drug product which is clearly undesirable. Various optical and electron microscopy techniques have been applied to this problem along with several other analytical methodologies [2]. There is an expectation by regulatory authorities that pharmaceutical manufacturers will understand the propensity of their product to cause corrosion and to monitor corrosion on accelerated stability studies [3]. Evaluation of incoming and treated (washing followed by depyrogenation) vials before filling with drug product is a key element of these glass corrosion evaluations. These initial studies serve as the standard against which progressive glass corrosion will be judged.

Two of the more common microscopy techniques applied to glass vial interior surface studies are Differential Interference Contrast (DIC) optical microscopy and Scanning Electron Microscopy (SEM). DIC has the advantages of examination of the whole vial with minimal sample preparation whereas SEM has the advantage of high resolution images even though the glass vial must be sectioned for analysis. In practice, DIC is used to locate possible regions of corrosion or of glass imperfections in the vial followed by breaking or cutting the glass in these regions and mounting on SEM stubs for examination. For tubular glass vials, the neck and heel of the vial are the most vulnerable regions for corrosion due to the method of vial manufacture. The manufacturing process can result in various kinds of glass imperfections including pits and divots. These imperfections can be interpreted as glass corrosion in stability studies unless incoming vials are well characterized.

Figures 1a and 1b show DIC images of the heel region of two different vial types before cleaning and filling. Pits and other interior glass imperfections can be seen clearly in the microscope, although difficult to photograph well. Figure 2a and 2b also show SEM images of imperfections from two different vial types before cleaning and filling. Figure 2A shows low level pitting while Figure 2B shows a more severe level of pitting and surface imperfections. In product stability trials, imperfections associated with Figure 2A often show little change over many $(>24)$ months at accelerated temperature and humidity conditions. Imperfections associated with Figure 2B, however, often lead to evidence of progressive corrosion in only a few months stability storage. Without thorough examination of incoming vials, the evidence of progressive corrosion may implicate the drug product whereas the glass itself may be the main factor.

Finally, it should be noted that microscopy is one part of a larger study of glass corrosion. The filled vials need to be examined for the presence of particles and the solution itself should be examined for dissolved glass elements ( $\mathrm{Si}$, etc.) by elemental analysis techniques such as inductively coupled plasma spectroscopy (ICP) before a judgment of progressive glass corrosion can be rendered. 


\section{REFERENCES}

[1] Iacocca R.G., Allgeier A., J Mater Sci 42, 2007 pp. 801-811.

[2] Carlton R.A., Micros \& Microanal 19, Suppl 2, 2013 pp 284-285.

[3] United States Pharmacopeia, Chapters 1660 and 660, 2014
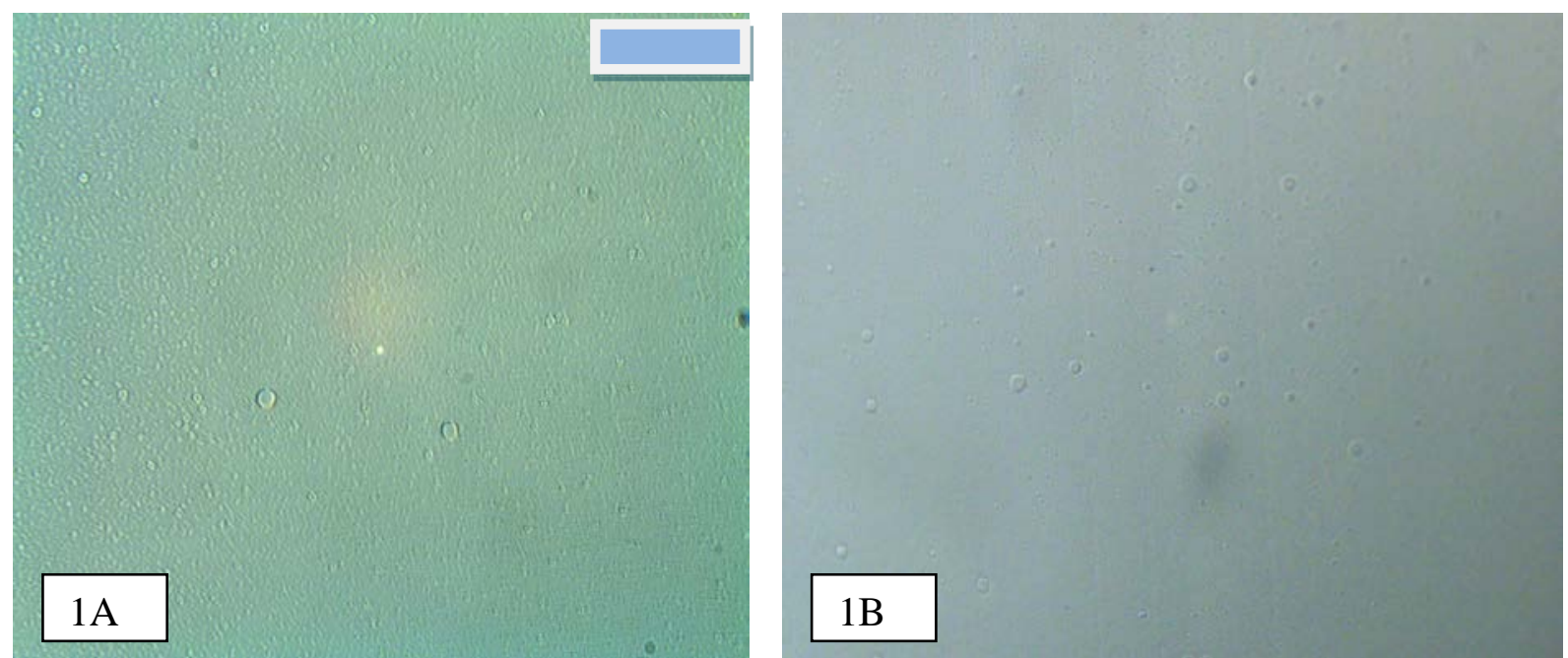

Fig. 1 DIC Images of Initial, Unfilled Glass Vials Image Size $=720$ x $500 \mu \mathrm{m}$
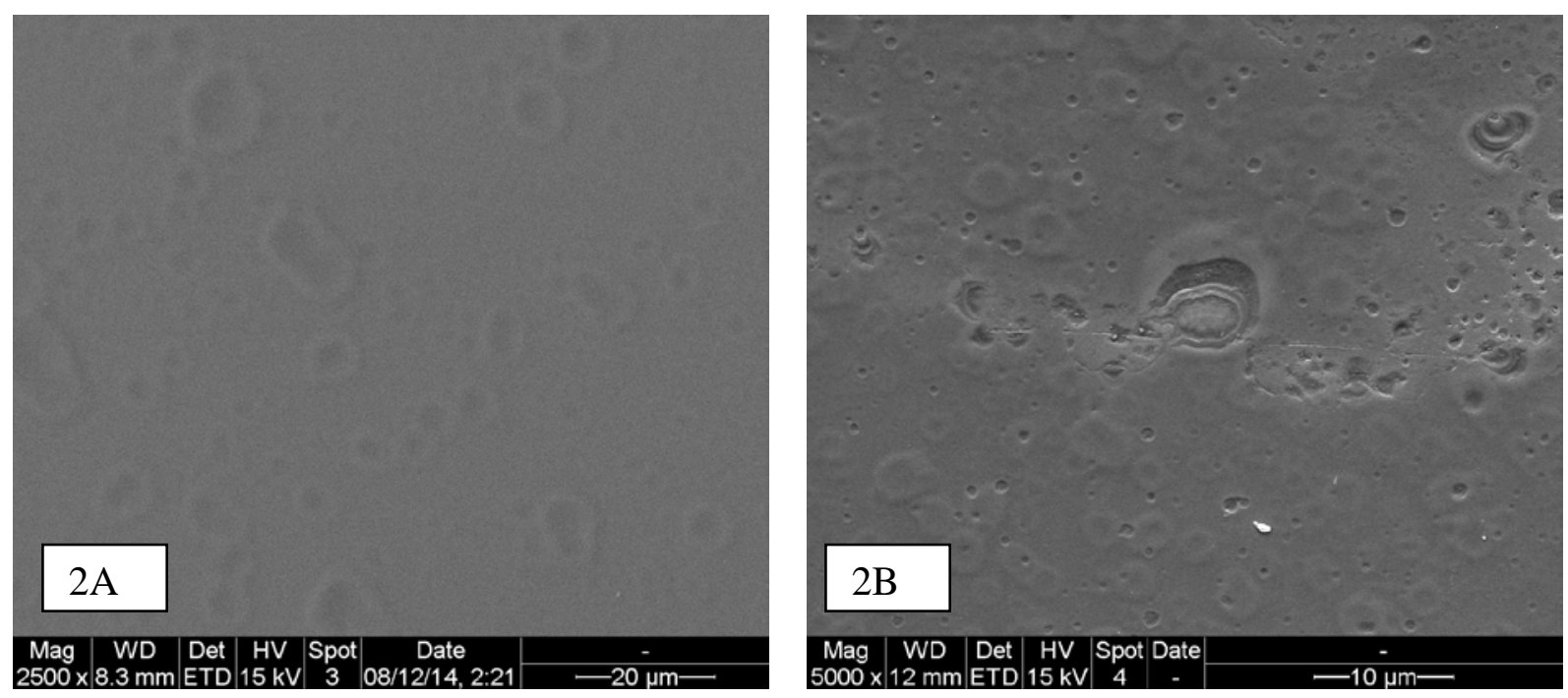

Fig. 2 SEM Images of Initial, Unfilled Glass Vials 